\title{
Bottom-Up-Feedback als Form der Partizipation: Hilfreiche Rückspiegelung, Risiko oder verschwendete Zeit?
}

\author{
Kerstin Zlöbl'1 \\ Online publiziert: 29. Juli 2019 \\ (c) Der/die Autor(en) 2019
}

\section{Zusammenfassung}

In Zeiten zunehmenden Wandels und steigender Informationstransparenz erhöhen sich die Anforderungen von Personen an Organisationen und sie werden in der Äußerung ihrer Bedürfnisse mündiger. Organisationen, die sich der Bedeutung ihrer Mitglieder für den nachhaltigen Organisationserfolg bewusst sind, reagieren auf diese Entwicklungen mit der Implementierung unterschiedlicher Kommunikationsformate, die darauf abzielen, die Partizipation der Organisationsmitglieder an der organisatorischen Entwicklung sicherzustellen. Demokratisierende Ansätze eröffnen jedoch ein Spannungsfeld in hierarchisch ausgerichteten Organisationssystemen, die von organisationalen Abhängigkeiten und individuellen Zugehörigkeiten geprägt sind. Daher benötigen sie tragende Strukturen sowie das Commitment der Beteiligten, um einen nachhaltigen Mehrwert zu liefern. Bottom-Up-Feedback ermöglicht es, Hintergründe von Handlungen und Bedürfnissen der Organisationsmitglieder zu erfahren. Mit Fokus auf ein aktuelles empirisches Forschungsprojekt diskutiert dieser Beitrag der Zeitschrift Gruppe. Interaktion. Organisation. Bottom-Up-Feedback als Form der Partizipation in Organisationen, wobei Herausforderungen und Prämissen für die Etablierung solcher Feedbackprozesse diskutiert werden.

Schlüsselwörter Bottom-Up-Feedback · Feedback · Kommunikation $\cdot$ Partizipation $\cdot$ Hierarchie

\section{Bottom-Up-Feedback as a form of participation: Helpful reflection, risk or wasted time?}

\begin{abstract}
In times of increasing change and information transparency requirements of individuals increase and they become more mature in addressing their needs. Organizations enhance the involvement of their members in organizational processes through the implementation of participative communication formats. However, democratic formats of communication open a field of tension in fundamentally hierarchically aligned organizations, because there are dependencies and individual affiliations, which could restrain communication. Moreover, to create value out of the processes grounded structures and the commitment of the workforce are necessary. Bottom-Up-Feedback delivers an opportunity to get insights on the reasons of workforce requirements. Relating to an actual research project this contribution to the journal Gruppe. Interaktion. Organisation. investigates Bottom-Up-Feedback as an opportunity for participation in organizations and discusses challenges and premises for the successful implementation of Bottom-Up-Feedback processes.
\end{abstract}

Keywords Bottom-Up-Feedback $\cdot$ Feedback $\cdot$ Communication $\cdot$ Participation $\cdot$ Hierarchy

\section{Problemstellung}

Die rasche Entwicklung von Schwellenländern, die Digitalisierung von Kommunikationstechnologien und technologische Innovationen sowie ihre Auswirkungen auf die Ab-

Kerstin Zlöbl, BA, MA

Kerstin.zloebl@hotmail.com

1 Alpen Adria Universität Klagenfurt, 9020 Klagenfurt/Kärnten, Österreich läufe von Arbeitsprozessen zwingen Organisationen dazu, sich von traditionellen Strukturierungsprinzipien und strikt hierarchischen Ordnungen zu verabschieden und ihr Organisationsdesign neu zu überdenken (Nagel 2014, S. 4). Das 
Organisationsdesign stellt ein Regelwerk bzw. eine Orientierung für die erfolgreiche Bewältigung von neuen Herausforderungen dar. Zum einen beinhaltet es die hierarchische Ordnung, die Strukturen der Zusammenarbeit innerhalb der Organisation festlegt und Orientierung über die Hierarchieebenen des Unternehmens gibt. Zum anderen - weit relevanteren Punkt - umfasst es Faktoren, die auf die dort arbeitenden Menschen einwirken. Diese sind beispielsweise Umweltbedingungen, Kommunikationsmuster, Geschäftsprozesse, Anforderungen an Arbeitsstellen, Skills der MitarbeiterInnen, die Wettbewerbssituation, Arbeitsbedingungen sowie Führungsstrukturen und -systeme (Nagel 2014, S. 3 ff.). Letzteres verändert sich insofern rasant, als dass die Mündigkeit von Organisationsmitgliedern steigt und sich ihre Anforderungen an Organisationen als Arbeitergeber erhöhen.

Organisationsmitglieder sind die ausführenden Kräfte von Handlungen, die für die Erfüllung des Organisationszweckes notwendig sind. Ihr Einfluss auf Entscheidungsund Entwicklungsprozesse ist maßgeblich, weshalb die Mitglieder vieler Organisationen wertvollstes Gut sind. Vor diesem Hintergrund ist ihre Gefolgschaft für Organisationen unerlässlich, um handlungsfähig zu bleiben. Diesem Systeminteresse geschuldet rücken sie und ihre Interessen bereits seit dem Anbeginn gruppendynamischer Forschung ins Licht und wurden als „organisationale Vorgänge“ zum Untersuchungsgegenstand (Krainz 2011).

Dabei fokussieren Organisationen nicht ohne Hintergedanken die Interessen ihrer Mitglieder und agieren keineswegs von reiner Nächstenliebe getrieben, wenngleich die Interventionen auch für MitarbeiterInnen Vorteile aufzeigen: Beispielsweise vermittelt das Interesse an den Bedürfnissen der Mitglieder ihnen gegenüber ein Gefühl von Mitsprache und Wertschätzung. Seitens der Organisationen verfolgt es jedoch auch den Zweck, Informationen zu der Steuerung von Organisationsmitgliedern zu erlangen. Ähnlich zwielichtige Zwecke können auch partizipative Ansätze verfolgen: Einerseits erinnern sie an einen Befreiungsschlag gegen die frühere ,Sklaverei“ in hierarchischen Systemen, in denen Organisationsmitglieder nur engstirnig und ohne Berücksichtigung ihres eigenen Willens Aufgaben auszuführen hatten. Die Intention moderner Partizipationsformen, die zumeist auch unter einer ,Demokratisierung der Arbeitswelt" diskutiert werden, ist dabei ein Bild von Wertschätzung und Kooperation zwischen Hierarchieebenen zu vermitteln, in dem MitarbeiterInnen Mitsprache eingeräumt wird. Durch die Identifikation ihrer Interessen und Einstellungen wird ein Gefühl von Wertigkeit und Mitsprache transportiert. Denn aufgrund der gestiegenen Anforderungen der Mitglieder reicht es für Organisationen oft nicht mehr aus, lediglich transparent zu delegieren und auf blinde Gefolgschaft zu hoffen. Vielmehr stehen Organisationen auch in Abhängigkeit zu ihren Mitgliedern, die Widerstände leisten, wenn Stimmen unterdrückt werden und die Belegschaft sich nicht ausreichend ,gehört" fühlt. Dem wirken partizipative Formate entgegen. Andererseits (und weniger populär angepriesen) ermöglichen partizipative Ansätze Organisationen die Erlangung von sensiblen Informationen über Einstellungen und Bedürfnisse der Organisationsmitglieder. Dadurch wird aber auch ihre gezielte Beeinflussung und Steuerung im organisationalen Interesse ermöglicht. Dies ist insofern kritisch $\mathrm{zu}$ sehen, da Wissen über die Bedürfnisse von Organisationsmitgliedern für deren Manipulation genützt werden kann. So werden die Interessen von MitarbeiterInnen als Anreize für deren Beeinflussung herangezogen, um sie zur Gefolgschaft zu bewegen, wofür wiederum partizipative Kommunikationsprozesse genützt werden. Folglich benötigen Organisationen und sich darin befindende Führungssysteme geeignete Partizipationsformate, welche die Bedürfnisse von MitarbeiterInnen identifizieren und berücksichtigen. Zur Erfüllung dieses Zweckes dienen unterschiedliche Kommunikationsinstrumente dem Austausch zwischen Organisationsmitgliedern. Eines dieser Instrumente ist Feedback.

Wird dabei die Erhebung der Interessen von geführten MitarbeiterInnen fokussiert, eignet sich vor allem das Format Bottom-Up-Feedback, bei dem Rückmeldungen seitens der Belegschaft an ihre Vorgesetzten adressiert werden. Durch den Austausch zwischen Organisationsmitgliedern werden Informationen über ihre Meinungen und $\mathrm{Be}$ dürfnisse erlangt. Doch während Top-Down-Feedbackprozesse (d.h. Rückmeldungen von hierarchisch Höhergestellten an ihnen unterstellte MitarbeiterInnen) im Arbeitsalltag allgegenwärtig sind, finden Bottom-Up-Feedbackprozesse durch MitarbeiterInnen (d.h. Feedback an hierarchisch Höhergestellte) nur selten statt und sind in ihrem Outcome in der Praxis oft umstritten. Vielfach wird die Effizienz in Frage gestellt, da die Offenheit bei der Äußerung von Meinungen durch hierarchisch abhängige MitarbeiterInnen weder erzwungen noch überprüft werden kann. Neben der Etablierung tragender, struktureller Regelungen für die Gewährleistung eines vertraulichen Prozesses benötigt es für Offenheit in Feedbackprozessen das Commitment aller Beteiligten, das durch unterschiedliche Prämissen beeinflusst wird. Zum einen könnten Organisationsmitglieder aufgrund ihrer hierarchischen Abhängigkeit von den FeedbacknehmerInnen Konsequenzen befürchten, sofern Rückmeldungen nicht anonym durchgeführt, sondern öffentlich (z.B. in persönlichen Gesprächen) adressiert werden. Auch wenn eine grundsätzliche Bereitschaft zur Partizipation und eine offene Haltung für kritische Rückmeldungen gegeben wäre, werden Aussagen von dem Bewusstsein über hierarchische Abhängigkeiten beeinflusst. Zum anderen birgt das Feedback auch für Führungskräfte das Risiko des Autoritätsverlustes aufgrund der Bewertungen ihrer MitarbeiterInnen. Denn in Rückmeldungen zu Führungshaltungen durch 
die Belegschaft werden neben fachlichen Inhalten auch verhaltensbezogene Rückschlüsse ableitbar, die individuelle sowie organisationale Bewertungen betreffen können und die Notwendigkeit zu Veränderungen aufzeigen, welche im Alltag sonst nicht von unterstellten Organisationsmitgliedern eingeholt werden würden. Partizipative Kommunikationsformate fokussieren somit vermehrt die Aufweichung hierarchischer Grenzen - und bedeuten Veränderung, möglicherweise Machtverlust auf Seiten der Vorgesetzten und mehr Autonomie auf Seiten der MitarbeiterInnen. Daher bringen Formen der Partizipation nicht nur Chancen, sondern stets auch Risiken mit sich.

Organisationen sind - wenn auch oftmals nicht bewusst nach außen gekehrt - hierarchisch aufgestellt und nicht dazu bestimmt demokratisch zu agieren (Kühl 2011, S. 19). Daher benötigt die Implementierung partizipativer Kommunikationsformate die Akzeptanz der Beteiligten aber auch tragende Strukturen und Regelungen, um einen vertraulichen Prozess zu gewährleisten und Feedback verwerten zu können. Vor diesem Hintergrund bildet das Instrument Feedback eine interessante Möglichkeit, um organisationale Lernerfahrungen unter Einbezug der Betroffenen zu sichern, Wissen zu transferieren und Kommunikation sicherzustellen. Seine Anwendung in Form von Bottom-UpFeedback erweist sich einerseits als besonders geeignet, um einen Beitrag zur „Demokratisierung“ von Organisationen $\mathrm{zu}$ leisten und eine partizipative Organisationskultur zu etablieren, impliziert andererseits jedoch auch Gefahren und Risiken des Fehlschlagens, bedingt durch die hierarchische Ausrichtung, der Organisationen unterliegen.

Vor diesem Hintergrund nimmt sich der Beitrag zunächst theoretischen Grundlagen zur Bedeutung von Kommunikation und Feedback in Organisationen an und diskutiert im zweiten Schritt ein empirisches Forschungsprojekt (Zlöbl in Vorbereitung), das sich der Erforschung von Bottom-UpFeedback angenommen hat. Im dritten Teil des Beitrages wird ein Fazit aus den Erkenntnissen des Forschungsprojektes abgeleitet, das Herausforderungen und notwendige Prämissen für die Implementierung von Bottom-Up-Feedbackprozessen für eine erfolgreiche Anwendung in der Praxis erläutert.

\section{Zur Bedeutung von Kommunikation und Feedback in Organisationen}

Organisationen dienen primär der Erfüllung eines bestimmten (oft wirtschaftlichen) Zweckes und sind dabei hierarchisch ausgerichtet (Kühl 2011, S. 18f.). Um als funktionale Systeme dienlich sein zu können, haben sie bestimmte Auflagen und Anforderungen $\mathrm{zu}$ erfüllen, die von unterschiedlichen Seiten an sie gestellt werden. Begleitet werden diese Anforderungen durch technologischen Wandel, steigende Informationstransparenz, die rasche Verbreitung von Wissen sowie durch Organisationsmitglieder, die individuelle Bedürfnisse haben (und diese auch artikulieren möchten), deren Erfüllung für den Erhalt der Leistungsfähigkeit notwendig sind.

Egal ob im privaten oder beruflichen Leben, Menschen sind stets mit Organisationen konfrontiert und involviert. Deshalb werden Organisationen vielmals als organisch und lebend definiert, da sich Personen darin befinden, die menschliche Empfindungen verspüren. Diese wirken sich auf ihre Aktionen und Reaktionen aus, wodurch das Geschehen in Organisationen beeinflusst wird (Krainz 2011, S. $122 \mathrm{ff}$.). Seit längerem ist bekannt, dass Organisationsmitglieder zwar als Arbeitskräfte gesehen werden, die Beiträge zu der Erfüllung der Organisationsziele leisten, jedoch stets auch individuelle Bedürfnisse und Interessen verfolgen, die von denen der Organisationen abweichen (Krainz 2011, S. 125). Bereits in den 1930er Jahren wurde die Dynamik von Gruppen als Einflussfaktor auf die Leistungserbringung von Organisationsmitgliedern zum Fokus und durch diverse Experimente und Forschungen in der damaligen „Human-Relations-Bewegung“ bestätigt. Sie widmete sich dem Faktor Mensch in Organisationen und fokussierte seine Beziehungen innerhalb der organisationalen Umwelt. Dabei wurden Zusammenhänge zwischen dem erfolgreichen Funktionieren von Organisationen und der Zufriedenheit der sich darin befindenden Individuen festgestellt. Letzteres wird unter anderem von Interaktionsprozessen beeinflusst. Diese können formale Fachgespräche, informelle Café-Treffen und sämtliche andere Interaktionsprozesse sein, in denen Emotionen von Organisationsmitgliedern offengelegt werden, welche die Ausführung der Arbeitsaufgaben unmittelbar oder mittelbar betreffen (Krainz 2011, S. 144 ff.). Organisationale Kommunikationsprozesse beinhalten Berichtwege, Leitlinien, Handlungsmuster und Spielregeln der Organisationen und legen fest wer mit wem kommuniziert, wer welche Informationen erhält, wie diese gewichtet werden und welche Glaubenssätze verfolgt werden. Sie verändern sich laut Simon (2002, S. 7 ff.) selbstorganisiert durch Veränderungen ihrer Umwelten. Organisationsmitgliedern bieten sie einen Kommunikationsrahmen, da sie in ihren Köpfen Wissen rund um die Organisation und darin stattfindende Prozesse (re)produzieren. Dabei werden organisationsinterne aber auch aus ihrer externen Umwelt stammende Informationen und Daten aufgegriffen und durch Interaktionen der Organisationsmitglieder zu nützlichem Organisationswissen transferiert, was zur Weiterentwicklung organisationaler Abläufe beitragen kann (Wehling 2007, S. 208). Um Diskussionsprozesse $\mathrm{zu}$ etablieren, Wissen $\mathrm{zu}$ (re)produzieren und Informationen über Organisationsmitglieder zu erlangen, benötigt es zum einen eine kooperative Organisationskultur, die Gruppenkommunikation zulässt und zum 
anderen Rollen in Gruppen, die solche Austauschformate wahrnehmen (Krainz 2011, S. 143).

Organisationen sind sich ihrer zunehmenden Komplexität und der steigenden Autonomie ihrer Mitglieder bewusst (Mirow und Matzler 2012, S. 35). Daher gewinnt ein Organisationsdesign an Bedeutung, das hierarchische Strukturen durch Kooperationsmodelle zwischen Organisationseinheiten aufweicht. Dieser Netzwerkaufbau bringt den Vorteil, gemeinsam rasch auf entstehende Veränderungen eingehen zu können und Organisationen am Markt zu stärken (Nagel 2014, S. 4). Die Dezentralisierung von Wissen in unterschiedliche Organisationseinheiten erfordert neue Kommunikationsmodelle über Hierarchieebenen hinweg. Vor diesem Hintergrund werden auch klassisch hierarchische Systeme getrieben, Informationen durch Kommunikationsprozesse von unteren Hierarchieebenen einzuholen, damit der Einbezug relevanter Fakten und Informationen von allen Seiten sichergestellt werden kann. Dadurch soll der zunehmenden Komplexität organisationaler Umwelten durch Transparenz entgegengewirkt und auf die steigende Mündigkeit von Organisationsmitgliedern durch ihre Partizipation an Prozessen reagiert werden.

Dies bedeutet jedoch keine propagierte Aufhebung hierarchischer Ordnungen in Organisationen, welche für deren Überleben durchaus Sinn machen (von Ameln und Heintel 2016, S. 9). Jedoch zielen partizipative Ansätze auf die Etablierung und Akzeptanz einer kooperativ ausgerichteten Organisationskultur ab, welche die hierarchische Ordnung schwächt, weil sie durch hierarchieübergreifende Mitbestimmungsmöglichkeiten angreifbar wird und Kommunikationsprozesse demokratisierend wirken können. Denn durch die Einrichtung von partizipativen Kommunikationsmodellen werden MitarbeiterInnen in organisationale Entwicklungsprozesse einbezogen und ihnen ein Mitbestimmungsrecht eingeräumt.

Auch wenn demokratische Ansätze in Form von partizipativen Kommunikationsprozessen keine totale Bestimmung durch MitarbeiterInnen ermöglichen, bieten sie jedoch die Möglichkeit Rückschlüsse darüber zu ziehen, ob sie ihre Führungskräfte akzeptieren oder ablehnen. Daher ist ihr Erfolg wesentlich von dem Commitment der Beteiligten abhängig, was antizipativ nicht immer gegeben ist, denn Demokratie bringt nicht nur Möglichkeiten, sondern geht auch mit Risiken und Herausforderungen einher. Sie bedeutet für Führungskräfte einen Machtverlust, erfordert Selbstautonomie der MitarbeiterInnen sowie die Übernahme von Verantwortung für ihre Handlungen und Aussagen. Während es früher gänzlich unüblich war, Rückmeldungen an Vorgesetzte zu adressieren oder ihnen gar kritisch zu widersprechen, erleiden obere Hierarchieebenen und Führungssysteme durch Partizipationsprozesse heutzutage nahezu einen Identitätsverlust: Sie werden vielfach als Anlaufstelle für sämtliche Belange der Organisationsmit- glieder gesehen und Unterstützung von ihnen wird erwartet. Durch die Verschiebung der Anforderungen an Führung und MitarbeiterInnen in partizipativen Formaten ändern sich auch vorherrschende Leistungsrollen, Zugehörigkeiten und Identitäten. Zum Ausdruck gebracht werden die Anforderungen in Diskussionsrunden, Austauschrunden aber auch Vier-Augen-Gesprächen. Vielfach geschieht dies im Verlauf der Gespräche auch unbewusst. Daher eignen sich eigens dafür konzipierte Formate, um Interaktionen und deren Wirkungen zu analysieren und rückzuspiegeln:

In der Metakommunikation und durch Feedback werden Diskussionsprozesse und deren Wirkungen reflektiert (Lang 2003, S. 49). Dabei rücken Individuen und ihre Handlungsweisen in den Vordergrund und erhalten Rückmeldungen zu ihrem Wirken in gewissen Situationen. Die Rückmeldungen basieren auf den subjektiv gebildeten Meinungen der feedbackgebenden Personen, die Handlungen sowohl sachlich als auch emotional bewerten: „Die Meinung ist die Bewertung von Information, in ihr vermischt sich das Persönlich-emotionale mit dem Sachlich-dienstlichen“ (Krainz 2011, S. 172). Rückmeldungen sind daher stets von persönlichen Emotionen der FeedbackgeberInnen begleitet, die Aufschluss über ihre Gefühlslage geben. Ihre Emotionslage beeinträchtigt ihre Wahrnehmung und kann sich in weiterer Folge auch auf ihre Arbeitsleistung auswirken, da diese von Emotionen beeinflusst wird. Basierend auf der Erkenntnis über die Abhängigkeit von Emotionen und Arbeitsleistungen sind die Meinungen von Organisationsmitgliedern für Führung und Organisationsentwicklung relevant, da sie Einfluss auf die organisationale Entwicklung nehmen und Führungskräfte durch deren Kenntnis Einfluss auf MitarbeiterInnen ausüben können. Im Rahmen der Identifizierung und Analyse von Stimmungen und Meinungen der Organisationsmitglieder erlangte dadurch im Laufe der Zeit Bottom-Up-Feedback an Bedeutung, da es die Sichtweisen der MitarbeiterInnen fokussiert und einfängt, wodurch Rückschlüsse auf ihre Emotionslagen gezogen werden können.

Denn Emotionslagen entstehen durch Eindrücke und Wahrnehmungen von Personen und beeinflussen sie in ihrem Handeln sowohl in der privaten als auch der beruflichen Laufbahn. Eindrücke und Wahrnehmungen werden beständig gebildet und häufig geäußert (Schuler 2004, S. 25). Feedback bedeutet die Rückspiegelung dieser gewonnenen Eindrücke und gebildeten Meinungen und sollte unmittelbar gegeben werden (Sarges 2013, S. 861). Wahrnehmungen von Verhaltensweisen werden immer mit individuellen Emotionen verbunden. Bestehende Gefühle nicht zu äußern kann vor allem bei negativen Eindrücken die Beziehung zwischen betroffenen Personen stören. Werden negative Emotionen lediglich indirekt (z.B. durch Ärger, Unmut) zum Ausdruck gebracht, ist für EmpfängerInnen deren Ursprung meist nicht nachvollziehbar, wodurch Interventionen zu der Veränderung des Zustandes erschwert 
werden. Denn um einen gewissen Ist-Stand beizubehalten oder wiederherzustellen ist es notwendig, dem Gegenüber die eigene Gefühlslage bewusst zu machen (Lang 2003). Die namentliche Bezeichnung des Gespräches, das die Rückmeldung von Eindrücken zum Inhalt hat, ist dabei eher sekundär und unterschiedlich, verfolgt jedoch meist die selbe Absicht der Mitteilung von Meinungen. Ob das Gespräch als Feedback, Evaluation, Rückmeldung oder Beurteilung tituliert wird, ist in der Praxis oft irrelevant. So lassen sich in Organisationen beispielsweise Aktivitäten registrieren, die zwar alle unterschiedlich heißen, letztlich aber dennoch auf dasselbe abzielen - das Einholen von Meinungen der Organisationsmitglieder. Beschäftigt man sich näher mit dem Feedback-Begriff, finden sich jedoch gewisse Abgrenzungen. Ditton und Müller (2014, S. 11 ff.) beispielsweise grenzen Feedback klar von dem Begriff Rückmeldung ab, da Feedback verhaltens- und zeitnäher und personenbezogen sei, wogegen Rückmeldungen verhaltens- und zeitferner seien und einen Systembezug aufweisen. Evaluationen hingegen werden als Prozesse angesehen, die Feedbacks enthalten, ebenso wie Beurteilungen. Feedback umfasst also mehrere Begriffe, je nach Format und den Absichten, die mit den Meinungsäußerungen verfolgt werden, kann es jedoch unterschiedlich bezeichnet werden (Ditton und Müller 2014, S. $11 \mathrm{ff}$.).

$\mathrm{Da}$ in dem vorliegenden Artikel Bottom-Up-Feedback in Organisationen als mögliche Form der Partizipation untersucht wird, liegt der Fokus klar auf den inhaltlichen und emotionalen Aspekten der SenderInnen und EmpfängerInnen sowie deren Hintergrundgedanken. Fokussiert werden dabei vor allem Treiber und Hemmnisse für Bottom-UpFeedback, da sie im Rahmen des aktuellen Diskurses über eine Demokratisierung von Organisationen von besonderem Interesse sein könnten, wenn Feedback von MitarbeiterInnen als Form der Partizipation in Organisationen genützt werden soll. Die Etablierung dieses Prozesses scheint jedoch mit wesentlich mehr Herausforderungen und schwierigeren Begleiterscheinungen verbunden zu sein als es bei klassischem Top-Down-Feedback der Fall ist, was der hierarchischen Prägung von Organisationen geschuldet ist und viele Organisationsmitglieder davor hemmt, Rückmeldungen offen zu adressieren.

\section{Empirisches Anschauungsbeispiel}

Um die theoretischen Überlegungen empirisch zu untermauern werden in weiterer Folge die Ergebnisse eines Forschungsprojektes (Zlöbl in Vorbereitung) dargelegt, das sich mit den Themen Führung und Bottom-Up-Feedback in Hinblick auf Herausforderungen und notwendige Prämissen für dessen erfolgreiche Implementierung in vorherrschenden Führungssystemen beschäftigt. Untersucht wurden da- bei öffentlich rechtliche sowie wirtschaftliche Organisationen. Im Rahmen der empirischen Untersuchung wurden neben Beobachtungen und narrativen Interviews mit Führungskräften auch MitarbeiterInnen mittels Gruppendiskussionsverfahren interviewt und zu ihren Meinungen und Erfahrungen mit partizipativen Feedbackformaten befragt (insgesamt 19 Führungskräfte und acht Gruppen von MitarbeiterInnen bestehend aus bis zu fünf Personen). In Anlehnung an die theoretischen Überlegungen zur Grundaxiomatik der Interventionsforschung (Heintel 2005) wurde dem Feedbackgedanken auch im Zuge des Forschungsprozesses Rechnung getragen, da Daten nicht einfach nur erhoben wurden, sondern relevante Erkenntnisse, daraus resultierende Hintergrundtheorien und Schlussfolgerungen auch an die Beforschten rückgespiegelt wurden.

Da Bottom-Up-Feedback nicht nur für die empfangenden Führungskräfte, sondern auch für die sich äußernden MitarbeiterInnen eine Herausforderung darstellt, empfiehlt es sich, immer die Sichtweisen beider Seiten in die Forschung mitaufzunehmen. Im Folgenden soll daher ein Einblick in die Erfahrungsmuster der untersuchten Personen gegeben werden, die in Zusammenhang mit der Etablierung und Wirksamkeit von Bottom-Up-Feedbackformaten bedeutsam erscheinen.

\subsection{Unterschiedliche Führungs- und damit auch Feedbackverständnisse}

Vergleicht man die Aussagen aus den Interviews und Gruppendiskussionen wird schnell ersichtlich, dass Feedback abhängig von dem gelebtem Führungsverständnis ist und somit auch unterschiedliche Bedeutungen einnimmt. In dieser Hinsicht zeigt sich ein deutlicher Unterschied zwischen beiden Forschungsfeldern. Während das Datenmaterial von Führungskräften privatwirtschaftlicher Organisationen angelehnt an die definierten Führungsstile von Lewin et al. (1939, S. 273) vermehrt einen demokratischen Führungsansatz zeigt, in dem die laut Muir (2004, S. 13) definierten sozialen Kompetenzen wie das Eingehen auf die Bedürfnisse des Gegenübers bedeutsam sind, zeigt sich in öffentlich rechtlichen Organisationen ein eher klassisch autoritäres Führungsverständnis, das sich durch Kontrolle und Distanz definiert und vorsätzlich Leitungsaufgaben übernimmt. Folglich sind auch die Selbstbeschreibungen der Führungskräfte unterschiedlich. Während Führungskräfte in dem privatwirtschaftlichen Forschungsfeld laut eigenen Aussagen also als „Vorbilder, UnterstützerInnen und AnsprechpartnerInnen "1 agieren, identifizieren sich

\footnotetext{
${ }^{1}$ Die wörtlichen Zitate aus den Interviews und Gruppendiskussionen werden in weiterer Folge kursiv dargestellt und unter Anführungszeichen gesetzt.
} 
Führungskräfte des öffentlich rechtlichen Forschungsfelds unter anderem mit „Kontrolleuren“.

Diese unterschiedlichen Führungsverständnisse wirken sich auch auf die prinzipiellen Feedbackverständnisse in den Forschungsfeldern aus und spiegeln sich in Auffassungen von Führungskräften und MitarbeiterInnen wieder. So definieren InterviewpartnerInnen in Führungsfunktionen öffentlich rechtlicher Einrichtungen Feedback als „Mitteilung des Mitarbeiters über seine Arbeitsprozesse“, als „(...) eine konstruktive Kritik“, „,möglichst im gewissen Sinne natürlich auch eine Qualitätssicherung und Evaluierung, nicht". Geschuldet ist diese Einstellung nicht zuletzt dem vorherrschenden Hierarchiedenken öffentlicher Einrichtungen, dass zwar laut Aussagen der Führungskräfte im Laufe der Zeit abnimmt, aber immer noch vorherrscht und auch partizipative Kommunikationsformate beeinflusst. So wurde die Frage, ob sich Führungskräfte Feedback durch ihre MitarbeiterInnen einholen sogar mit „Nein, das mache ich nicht. Sollte man das?" beantwortet, was nicht zuletzt der Ansicht von Führungskräften geschuldet ist, dass ohnehin keine Rückmeldungen an Vorgesetzte in der Praxis adressiert werden würden: „Wenn sie dich fragen passt es oder passt es nicht oder? Sagen Sie das? Keiner oder? “. Im Unterschied dazu wird Feedback in den untersuchten privatwirtschaftlichen Organisationen breiter verstanden, als eine Art „angemessene Rückmeldung auf ein vorangegangenes Ereignis“. Was dabei als „Mitteilung“, „Arbeitsprozess“ oder ,angemessen“ gilt, wird von den GesprächspartnerInnen subjektiv definiert. Aus den unterschiedlichen Aussagen von Führungskräften und MitarbeiterInnen beider Forschungsfelder wird jedenfalls sichtbar, dass es keine einheitliche Definition für den Feedbackbegriff gibt. Aus Sichtweise der MitarbeiterInnen wird der Feedbackbegriff in beiden Forschungsfeldern vorwiegend mit Top-Down-Feedback und in den seltensten Fällen mit ihrer Möglichkeit, Rückmeldungen an Führungskräfte zu äußern, in Verbindung gesetzt. Vor diesem Hintergrund assoziieren sie Feedback damit, „dass es eigentlich nur Feedback gibt, wenn irgendwas gravierendes Schlechtes passiert ist“", „Nicht geschimpft ist gelobt genug, das ist bei uns die Devise“ und „kein Tadel ist Lob genug“. Dabei betonen sie, dass dieses negativ behaftete Feedbackverständnis sich durch die gesamte Organisation zieht.

Vor diesem Hintergrund wird deutlich, dass in den Forschungsfeldern zwar prinzipiell unterschiedliche Feedbackverständnisse seitens der Führungskräfte vorherrschen, MitarbeiterInnen beider Forschungsfelder Feedback jedoch primär mit „Top-Down-Feedback“ und Kritik assoziieren.

\subsection{Bottom-Up-Feedback in öffentlich rechtlichen Organisationen}

Folgt man den Ansichten einiger MitarbeiterInnen öffentlich rechtlicher Einrichtungen besteht ihrerseits kein explizites Bedürfnis nach partizipativen Kommunikationsformaten, in denen sie sich mitteilen können: „Also ich sehe jetzt zumindest für mich nicht die Notwendigkeit dazu. “ „Vor allem hat man ja gar nicht die Zeit immer für solche Sachen. “ „Ja wie gesagt, ich meine, dadurch das (...)/man sieht es ja laufend, wir sind eigentlich laufend im Gespräch, man bespricht eigentlich laufend Sachen." Aus der Grundzufriedenheit mit vorherrschenden, klaren Rollenabgrenzungen und beschränkten Kommunikationsmöglichkeiten geht zeitgleich das klare Anforderungsbild an Führungskräfte hervor, das vor allem „Orientierung“ von ihnen verlangt. (,Man hat immer das Gefühl, man kann nämlich zu jeden hingehen, fragen, eben auch zum Dr.X hingehen, wenn man eine Frage hat. (...) man bekommt vom Dr. X einfach immer/bekommst du gleich eine Antwort eigentlich.") Daher scheint die Rollenverteilung in den meisten Fällen sowohl für Führungskräfte als auch für MitarbeiterInnen akzeptabel und annehmbar. Weitaus schwieriger haben es MitarbeiterInnen öffentlich rechtlicher Einrichtungen, die sich Partizipation in Entscheidungsprozessen wünschen und ihre Meinungen kundtun möchten, jedoch keine Möglichkeiten dazu erhalten: InterviewpartnerInnen klagen etwa über ,Unmut “ und ,Streitereien “, als Reaktion auf deren fehlenden Einbezug und fehlende Transparenz in Entscheidungssituationen wird angemerkt: „Du kannst außer Sarkasmus und Trotzigsein gar nicht mehr anders reagieren, weil du dich fragst ja spinne ich jetzt oder". Seitens der Führungskräfte wird der oft vorherrschenden ,Jammerkultur “ aufgrund mangelnder Mitsprache mit der Begründung der zwingenden Einhaltung „strenger Gesetze“ und „strikter Vorgaben“ entgegnet, die Partizipationsmöglichkeiten begrenzt. Als Entschädigung dafür habe die Mitarbeit in öffentlich rechtlichen Einrichtungen laut Aussagen der Führungskräfte aber auch „,angenehme Begleiterscheinungen“, denn es sei auch für MitarbeiterInnen ein „Privileg in der Landesabteilung zu arbeiten".

Inwiefern das Privileg einer gewissen Arbeitsplatzsicherheit als Kompensation für fehlende Partizipationsmöglichkeiten von MitarbeiterInnen gelten gelassen wird, ist jedoch im Endeffekt von ihren Bedürfnissen abhängig.

\subsection{Bottom-Up-Feedback in privatwirtschaftlichen Organisationen}

Wie bereits aus dem eher breiten Feedbackverständnis privatwirtschaftlicher Organisationen kenntlich wurde, bestehen in privatwirtschaftlichen Organisationen mehr Möglichkeiten zu der Implementierung partizipativer Forma- 
te. Doch auch wenn sie vielfach seitens der Organisation angestoßen werden, entscheidet als Letztinstanz das vorherrschende Führungsverständnis über dessen zweckmäBige Implementierung. Auch wenn laut Führungskräften offene Gesprächskulturen dazu beitragen bei jeder Gelegenheit Bottom-Up-Feedback entgegenzunehmen und auch versucht wird ,in jedem Vertriebsgespräch oder auch in den Teamsitzungen auch immer, ein Feedback einzuholen ", so wird bei einer detaillierten Betrachtung des Datenmateriales ersichtlich, dass Führungsthemen vorzugsweise mit „Vertrauenspersonen“, „AssistentInnen“ oder „FührungskollegInnen " besprochen werden, da der Einbezug der gesamten Belegschaft aufgrund ihrer Größe offene Rückmeldungen hemmen würde. Eine Führungskraft beschreibt die Problematik von Feedback in großen Gruppen: „egal, was man sich da vornimmt, zum irgendwie eine Diskussion zu generieren oder ein Feedback einzuholen, das funktioniert nicht. Da steht man vorne wie so ein Lehrer vor der Präsentation, redet irgendwas und dann nicken alle und gehen raus und regen sich dann hinterher auf, ,ja-das müssen wir eh so akzeptieren “". Die Aussage unterstreicht, dass aufgrund der Gruppengröße und des Beiseins von Führungskräften offene Rückmeldungen zurückgehalten werden, auch wenn diese Unmut erzeugen. Daraus lässt sich ableiten, dass Bottom-Up-Feedback - sofern es zur Anwendung kommt - im Regelfall im kleinen Kreis wirksam stattfindet. Anders als im öffentlich rechtlichen Forschungsfeld dienen dafür laut Aussagen von Führungskräften und MitarbeiterInnen häufig ,halbprivate Rahmen“ wie „Dienstreisen“ oder „Teambuildings". Doch auch wenn die grundlegende Intention zu der Annahme von Rückmeldungen durch Führungskräfte besteht und angemessene Rahmen für Bottom-Up-Feedback vorhanden sind, wird die grundsätzliche Bereitschaft seitens der MitarbeiterInnen benötigt, diese Kommunikationsformate wahrzunehmen. MitarbeiterInnen begründen die Schwierigkeiten der Adressierung von Rückmeldungen an Führungskräfte dabei entweder mit distanzierten Verhältnissen zu Vorgesetzten (,das macht es uns auch schwierig, auf ihn zuzugehen") oder mit vorherrschender Unsicherheit über deren Reaktion auf die erhaltenen Rückmeldungen (,, weil wir auch teilweise nicht wissen, wie er das aufnimmt, wie er das händelt, wie er reagiert, wie dann diese Rückmeldungen, dieses Feedback ankommt, weil man hat in gewisser Weise eine ganz große Unsicherheit dabei "). Die Unsicherheit über die Reaktionen der Führungskräfte basieren zum einen auf der grundlegenden hierarchischen Abhängigkeit der MitarbeiterInnen zu ihren Vorgesetzten. Denn dadurch könnten aus Rückmeldungen Konsequenzen für sie resultieren. Zum anderen fürchten MitarbeiterInnen das Risiko der Gefährdung bestehender Beziehungsqualitäten durch die Äußerung von Bottom-Up-Feedback. Deshalb schwingen Überlegungen wie „nimmt er mir das dann persönlich, wenn ich ihm das sage oder das ist so, (...) stecke ich da so ein bisschen in der Zwickmühle da. Aber ich würde, also ich habe mir alles schon vorgenommen, das Mal irgendwann so zu sagen, weil das ist dann auch mal eine, ich sage mal eine Frage der Höflichkeit auch und der Ehrlichkeit, nicht. Dass man eben auch sagt, du, ich sehe da ein Problem und unter uns und schau mal, kannst du da was machen, kannst du da was ändern oder willst du das so weiterlaufen lassen? " mit, wenn das Thema Feedback an MitarbeiterInnen adressiert wird. Wenngleich man bei einer guten Beziehungsqualität von offenen Gesprächskulturen ausgeht, bestehen aufgrund der Nähe zu der jeweiligen Person auch Hemmungen bei der Adressierung von Rückmeldungen (,, Weil wenn man die Leute schon länger kennt oder das (...) macht es schwieriger. Oder wenn jetzt einer dabei ist ein halbes Jahr oder ein Jahr, dann hat man nicht so viele Hemmungen zum da direkter sein"). Wie aus den Aussagen hervorgeht, ist Bottom-UpFeedback deutlich von der Beziehungsqualität beeinflusst und wird im Regelfall nur dann seitens der MitarbeiterInnen initiiert, wenn laut ihren Aussagen grobe Unzufriedenheit über die „mangelnde Kommunikation“ oder ,wenig Verbindlichkeit" sowie der Wunsch besteht, dass Führungskräfte „Entscheidungen vielleicht nicht so übers Knie brechen " sollten. Diese geäußerte Unzufriedenheit lässt dabei auf den Wunsch nach mehr Partizipation rückschlieBen. Doch auch wenn MitarbeiterInnen ihre Empfindungen und Wahrnehmungen mitteilen, (oftmals auch lediglich an dritte Instanzen wie die Personalabteilung) wird dabei in den seltensten Fällen ein klarer Wunsch nach mehr Mitsprache gefordert. Führungskräfte müssen Rückmeldungen daher meist proaktiv als Feedback identifizieren und Handlungsbedarf daraus ableiten, was als schwierig beschrieben wird: „Also das ist das Schwerste daran Führungskraft zu sein, erstens kommt so eine Kritik (...) extrem selten. Wenn sie kommt, dann meistens eben durch die Blume. Und dann kommt sie immer, also ich bin der Meinung, dass ich das wahrscheinlich nicht einmal immer höre. Also zuerst muss ich es ja einmal mitbekommen ".

\section{Fazit}

Unter Berücksichtigung der Ergebnisse aus dem vorgestellten Forschungsprojekt wird zusammenfassend deutlich, dass Bottom-Up-Feedback zwar eine Möglichkeit der Partizipation von MitarbeiterInnen bietet, keineswegs jedoch ein Garant dafür ist. Der Erfolg der Partizipationsmaßnahme ist an bestimmte Bedingungen geknüpft. Das Datenmaterial zeigt in vielen Fällen bereits vorherrschende Prämissen, die für partizipative Ansätze ausschlaggebend sind: Ein kooperatives Führungsverständnis für die Bereitschaft von Führungskräften zu der Annahme von Feedback, den Willen von MitarbeiterInnen zu der offenen Äußerung 
von Rückmeldungen an ihre Vorgesetzten und Rahmen, in denen Rückmeldungen geäußert werden können.

\subsection{Kooperatives Führungsverständnis und Sensibilität für Feedback}

- Durch die Transparenz von Prozessen kann Führungskräften der Wert von Feedback durch MitarbeiterInnen für ihre individuelle Weiterentwicklung bewusst gemacht, sowie die Angst vor einem potentiellen Machtverlust genommen werden. Zudem wird eine Sensibilität für Feedback etabliert, denn Rückmeldungen werden im Arbeitsalltag von Führungskräften oft nicht wahrgenommen oder gehen unter. Dies liegt unter anderem auch darin begründet, dass die Weiterverwertung der Inhalte durch fehlende organisationale Strukturen erschwert wird, wodurch Bottom-Up-Feedback keine Verbindlichkeit erhält. Basierend auf dieser Erkenntnis wird ein Rahmen benötigt, in dem sie Feedback von MitarbeiterInnen einholen und wahrnehmen können. Dabei sollte seitens der Organisation Unterstützung bei der Weiterverwertung der Rückmeldungen geboten werden, ohne Führungskräften ein Gefühl der Überwachung zu vermitteln.

\subsection{Offenheit durch MitarbeiterInnen}

- Organisationsmitglieder benötigen Sicherheit und Rahmen, um offen konstruktive Rückmeldungen adressieren zu können. MitarbeiterInnen sind vielmals unsicher, in welcher Form und in welchem Rahmen sie Feedback angemessen an ihre Vorgesetzte adressieren können. Daher sollte eine Gesprächskultur etabliert werden, die regelmäßige Feedbackschleifen in Gesprächen mit Vorgesetzten vorsieht. Dabei zu beachten sind ihre Ängste des Verlustes bestimmter Beziehungsqualitäten sowie Unsicherheiten über potentielle Konsequenzen durch die Adressierung von Feedback. Die Kommunikation der Absichten von Feedback ist daher von besonderer Bedeutung, um MitarbeiterInnen von partizipativen Ansätzen zu überzeugen und ihnen Sicherheit für die offene Äußerung von Rückmeldungen zu vermitteln.

\subsection{Rahmen und Strukturen für Feedback}

- Auch wenn der Erfolg von Bottom-Up-Feedback letztendlich von den Inhalten der Rückmeldungen und von dem Umgang mit den Rückmeldungen abhängt, können Organisationen die Implementierung und Durchführung der Prozesse beeinflussen. Vorgegebene oder fehlende
Strukturen können Rückmeldungen fördern oder aber die Äußerung gewisser Themen hemmen. Organisationen sollten - wo notwendig und sinnvoll - Strukturen für Bottom-Up-Feedbackprozesse etablieren, welche die Offenheit der Beteiligten fördern. Feedbacksequenzen können im Rahmen der Regelkommunikation etabliert werden oder in separaten Treffen stattfinden. Beispielsweise schwächen informelle Austauschformate hierarchische Ordnungen und tragen zu der Verminderung von Unsicherheiten bei, weshalb MitarbeiterInnen dort eher Rückmeldungen adressieren. Organisationen sollten daher Möglichkeiten und Rahmen schaffen, in denen die bis dorthin informell laufenden Gespräche auch formalisiert werden können.

Erst das Bewusstsein und die Berücksichtigung dieser Gesichtspunkte ermöglicht die Implementierung von Bottom-Up-Feedback als demokratisierenden Prozess, der durch Rückmeldungen von MitarbeiterInnen zu der Weiterentwicklung von Führungssystemen und Organisationsprozessen genützt werden kann. Das dafür benötigte Commitment und die Bereitschaft zur Partizipation der Beteiligten bedeutet auf der Individualebene die Bereitschaft zu Veränderungen, die Übernahme von Verantwortung und die Motivation zu Kommunikation. In diesem Zusammenhang erhalten Organisationen die Möglichkeit, demokratisierende Kommunikationsformate durch die Bereitstellung von Rahmen und Formaten zu beeinflussen und durch tragende strukturelle Regelungen für die Sicherheit der Abläufe $\mathrm{zu}$ sorgen und dadurch Orientierung in den Prozessen $\mathrm{zu}$ bieten. Der dadurch entstehende Mehrwert liefert Erkenntnisgewinne für Führungskräfte und Organisationen, fördert die Motivation von MitarbeiterInnen durch Mitbestimmung und Wertschätzung und etabliert den Hauch eines demokratischen Gefühls in hierarchischen Organisationsgefügen.

Funding Open access funding provided by University of Klagenfurt.

Open Access Dieser Artikel wird unter der Creative Commons Namensnennung 4.0 International Lizenz (http://creativecommons.org/ licenses/by/4.0/deed.de) veröffentlicht, welche die Nutzung, Vervielfältigung, Bearbeitung, Verbreitung und Wiedergabe in jeglichem Medium und Format erlaubt, sofern Sie den/die ursprünglichen Autor(en) und die Quelle ordnungsgemäß nennen, einen Link zur Creative Commons Lizenz beifügen und angeben, ob Änderungen vorgenommen wurden.

\section{Literatur}

von Ameln, F., \& Heintel, P. (2016). Macht in Organisationen - Denkwerkzeuge für Führung, Beratung und Change Management. Stuttgart: Schäffer-Poeschel.

Ditton, H., \& Müller, A. (2014). Feedback: Begriff, Formen und Funktionen. In H. Ditton \& A. Müller (Hrsg.), Feedback und Rückmeldungen: Theoretische Grundlagen, empirische Befunde, prak- 
tische Anwendungsfelder (S. 11-29). Münster/New York: Waxmann.

Heintel, P. (2005). Zur Grundaxiomatik der Interventionsforschung. In P. Heintel, L. Krainer \& I. Paul-Horn (Hrsg.), WBI Klagenfurter Beiträge zur Interventionsforschung Bd. 1. Klagenfurt: AlpenAdria-Universität.

Krainz, E. (2011). Leiden an der Organisation. In K. M. Ratheiser, J. Menschik-Bendele, E. E. Krainz \& M. Burger (Hrsg.), Burnout und Prävention - Ein Lesebuch für Ärzte, Pfleger und Therapeuten (S. 115-197). Wien: Springer.

Kühl, S. (2011). Organisationen: Eine sehr kurze Einführung. Wiesbaden: VS.

Lang, A. (2003). Kommunikation. In Deutsche Versicherungsakademie DVA GmbH (Hrsg.), Kommunikation und Management Handlungsspezifische Qualifikationen 2. Aufl. Karlsruhe: Verlag Versicherungswirtschaft.

Lewin, K., Lippitt, R., \& White, R. K. (1939). Patterns of aggressive behavior in experimentally created "social climates". The Journal of Social Pschology, 10, 271-299.

Mirow, M., \& Matzler, K. (2012). Wie mächtig ist der Mächtige? Annäherung an das Phänomen der Macht aus der Sicht der Systemtheorie. In B. Knoblach, T. Oltmanns, I. Hajnal \& D. Fink (Hrsg.), Macht in Unternehmen - Der vergessene Faktor (S. 27-42). Wiesbaden: Gabler.

Muir, C. (2004). Learning soft skills at work. An interview with Annalee Luhmann. Business Communication Quarterly, 67(1), 95-101.

Nagel, R. (2014). Organisationsdesign - Modelle und Methoden für Berater und Entscheider. Stuttgart: Schäffer-Poeschel.

Sarges, W. (2013). Management-Diagnostik (4. Aufl.). Göttingen/ Bern/Wien/Paris/Oxford: Hogrefe.
Schuler, H. (2004). Beurteilung und Förderung beruflicher Leistung. Göttingen/Bern/Toronto/Seattle: Hogrefe.

Simon, F. (2002). Vorwort zur deutschen Ausgabe. In P. Holman \& T. Devane (Hrsg.), Change Handbook (S. 7-10). Heidelberg: Carl-Auer-Verlag.

Wehling, P. (2007). Kommunikation in Organisationen: Das Gerücht im organisationalen Wandlungsprozess. Wiesbaden: Deutscher Universitäts-Verlag I GWV Fachverlage GmbH.

Zlöbl, Kerstin (in Vorbereitung). Feedback an Führung - Hilfreiche Rückspiegelung oder verschwendete Zeit. Dissertation an der Alpen-Adria Universität Klagenfurt.

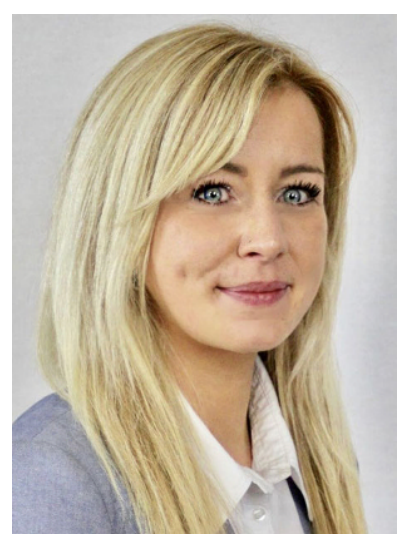

Kerstin Zlöbl widmet ihren Forschungsschwerpunkt nach einem betriebswirtschaftlichen Grundstudium den Themen Organisationsentwicklung und Gruppendynamik. Sie lebt in München und ist für eine Organisationsberatung im Bereich Technology Consulting tätig, in der sie ihre Arbeitsschwerpunkte auf den Bereich „Workforce Enablement" legt. 